\title{
Myocardial revascularization driven by functional testing and PET imaging
}

\author{
Pierre-Yves Marie, $M D, P h D,{ }^{a, b}$ and Alain Manrique, $M D, P h D^{c, d}$ \\ a CHRU-Nancy, Université de Lorraine, Nuclear Medicine \& Nancyclotep Experimental Platform, \\ Nancy, France \\ b Université de Lorraine, INSERM, UMR-1116, Nancy, France \\ c Department of Nuclear Medicine, CHU Cote de Nacre, Normandy University, Caen, France \\ d Normandie Univ, UNICAEN, Signalisation, Électrophysiologie et Imagerie des Lésions \\ d'ischémie-reperfusion Myocardique, FHU REMOD-VHF, Caen, France
}

Received Dec 2, 2019; accepted Dec 2, 2019

doi: 10.1007/s12350-019-01990-8

\section{See related article, pp. 1664-1672}

The study by Akil et al. presented in the present issue of the Journal of Nuclear Cardiology ${ }^{1}$ provides definite evidence that the initial presence of an abnormal flow reserve in a coronary territory constitutes a prerequisite for the further improvement of this flow reserve after myocardial revascularization. In other words, a significant improvement in flow reserve and thus, in myocardial perfusion, is unlikely in territories where coronary flow reserve is normal prior to revascularization.

This statement seems so obvious at first glance that it should already be unanimously shared in the medical community. However, even today, $35 \%$ of percutaneous revascularization procedures for a stable coronary disease may still be inappropriate or with uncertain indication with regard to that recommended by Heart Teams. $^{2}$

Thus, significant efforts are still required for reducing inappropriate indications of myocardial revascularization in patients exhibiting a stable and chronic heart disease. Many large studies and registries have demonstrated that the rate of cardiac events in patients with a stented stenosis is $2 \%$ to $3 \%$ per year and is definitely reduced compared to the $5 \%$ to $10 \%$ annual rate in patients with ischemic stenosis left untreated. ${ }^{3}$

\footnotetext{
Reprint requests: Pierre-Yves Marie, MD, PhD, CHRU-Nancy, Université de Lorraine, Nuclear Medicine \& Nancyclotep Experimental Platform, 54000 Nancy, France; py.marie@chru-nancy.fr J Nucl Cardiol 2021;28:1673-5.

$1071-3581 / \$ 34.00$

Copyright (C) 2019 American Society of Nuclear Cardiology.
}

However, it may be considered that stenting a non-ischemic coronary stenosis will not only be useless but also likely to worsen patient prognosis given the good prognosis associated with non-ischemic stenoses. One of the main issues is that a non-neglectable number of cardiologists still consider that a fully open artery is always more suitable than a narrowed artery, even if it does not lead to any significant change in the patient's symptoms, nor in the risk of heart attack or death. ${ }^{4}$ An additional issue is the low level of patient education and information, a great majority of these patients continuing to ask for aggressive treatments, being convinced that coronary revascularization will always help to prevent future attacks and prolong life. ${ }^{5}$

Furthermore, a high proportion of these stable patients did not undergo any functional testing prior to coronary angioplasty, ${ }^{6}$ contrary to that currently recommended in the US guidelines published 2 years ago in the Journal of Nuclear Cardiology, ${ }^{7}$ as well as in the recent European guidelines. ${ }^{3}$

A simple table, summarizing the recommended indications of myocardial revascularization in patients with stable angina or silent ischemia and extracted from the European guidelines, ${ }^{3}$ is reproduced herein in Table 1. This table has the advantage of being very simple, with the indications being listed independently of the method used for myocardial revascularization (i.e., angioplasty or bypass grafting). Briefly, myocardial revascularization may be indicated in patients with limiting symptoms on optimized medical therapy (angina or equivalent angina), as well as for certain angiographic patterns corresponding to high-risk coronary lesions for which an enhanced patient prognosis may be achieved by myocardial revascularization (i.e., $>50 \%$ stenosis of the left main or proximal left anterior 
Table 1. Indications for revascularization in patients with stable angina or silent ischemia (extracted from Ref. ${ }^{3}$ )

\section{Extent of CAD (anatomical and/or functional)}

\section{Class $^{\mathrm{a}}$ Level $^{\mathrm{b}}$}

\section{For prognosis}

Left main disease with stenosis $>50 \%^{\mathrm{c}}$

Proximal LAD stenosis $>50 \%{ }^{c}$

Two- or three-vessel disease with stenosis $>50 \%$ with impaired LV function (LVEF $\leq 35 \%$ )

Large area of ischemia detected by functional testing $(>10 \% \mathrm{LV})$ or abnormal invasive FFR $^{\mathrm{d}}$

Single remaining patent coronary artery with stenosis $>50 \%{ }^{c}$

For symptoms

Hemodynamically significant coronary stenosis in the presence of limiting angina or angina I

I A

I A

I A

I B

I C

equivalent with insufficient response to optimized medical therapy ${ }^{\mathbf{e}}$

$C A D$, coronary artery disease; $F F R$, fractional flow reserve; $i \omega F R$ instantaneous wave-free ratio; $L A D$ left anterior descending coronary artery; $L V$ left ventricular; $L V E F$ left ventricular ejection fraction

${ }^{a}$ Class of recommendation

${ }^{\mathrm{b}}$ Level of evidence

${ }^{c}$ With documented ischemia or a hemodynamically relevant lesion defined by FFR $\leq 0.80$ or iwFR $\leq 0.89$, or $>90 \%$ stenosis in a major coronary vessel

${ }^{\mathrm{d}}$ Based on FFR $<0.75$ indicating a prognostically relevant lesion (see Sect. 3.2.1.1)

e In consideration of patient compliance and wishes in relation to the intensity of anti-anginal therapy

descending artery, 2 or 3-vessel disease with left ventricular dysfunction, and single remaining patent coronary artery with stenosis $>50 \%$ ).

In all of these cases, however, the indication for revascularization needs to fulfill the additional criteria of a documented ischemia and/or a functionally relevant lesion at catheterization (see index $\mathrm{C}$ that is consistently added to each of these categories). This latter hemodynamic criterion, obtained in the catherization lab, is defined by low values of fractional flow reserve (FFR $\leq 0.80$ ) or of instantaneous wave-free ratio (iwFR $\leq 0.89$ ), while the sole anatomical criterion is that of a drastically narrowed artery with a $>90 \%$ diameter reduction. Indeed, only such $>90 \%$ stenoses are consistently associated with a FFR $\leq 0.80{ }^{8}$

Outside such cases of patients with limiting symptoms and/or high-risk coronary patterns, a myocardial revascularization may be still indicated although this still needs to fulfill the criterion of an even greater severe functional impairment, defined by the presence of a sufficiently large ischemic area, extended over more than $10 \%$ of the left ventricle, or of a FFR lower than 0.75 . These two criteria have already been considered as prognostically important, independently of patient symptoms and of coronary anatomy, albeit with a lower level of evidence than the other criteria (i.e., a B level, meaning that the supporting data for prognosis improvement are derived from only single randomized trials or non-randomized studies). ${ }^{3}$ Furthermore, very recent data from the large "Ischemia" randomized study, lead to consider that in patients with moderate-tosevere ischemia, the benefit of myocardial revascularization is mainly significant for quality of life parameters and not for death/myocardial infarction endpoints, contrary to that initially expected (more details are available through the following link: https:// www.acc.org/latest-in-cardiology/clinical-trials/2019/11 $/ 15 / 17 / 27 /$ ischemia). Hence, the characteristics of the ischemic patterns that need to be treated by myocardial revascularization deserve further investigations.

It may additionally be pointed out that non-invasive imaging is now recommended for first-line stress tests instead of exercise electrocardiogram (ECG) testing that suffers from too low sensitivity. ${ }^{3}$ In addition, PET could be the preferred first-line imaging test for patients unable to complete a diagnostic-level exercise stress imaging study, as recently stated through a joint position statement from the American Society of Nuclear Cardiology and of the Society of Nuclear Medicine and Molecular Imaging. ${ }^{9}$ Thus, the possibility of being easily associated with a physiological exercise test remains the main advantage of myocardial perfusion SPECT imaging.

Nevertheless, the PET technique, such as that used in the study by Akil et al. in the present issue of the $\mathrm{JNC},{ }^{1}$ is the gold standard for the noninvasive quantification of myocardial blood flow and flow reserve, even though there is some reported experience with MRI, Doppler echocardiography and more recently, with high-sensitivity CZT-SPECT cameras. ${ }^{10,11}$ In a 
relatively recent study, stress PET imaging was found to be more effective than conventional stress SPECT and cardiac CT angiography for identifying the coronary territories showing an abnormal FFR at catheterization. $^{12}$

Moreover, owing to PET imaging, the worse prognosis of patients with abnormal CFR has long been established, even in the absence of any significant stenosis of the epicardial coronary arteries. ${ }^{13}$ An abnormal CFR may indeed result from a mixture of vascular diseases, including microvascular abnormalities which are prevalent in patients with diabetes, chronic kidney disease and metabolic syndrome, as well as in patients with heart failure and preserved ejection fraction, atrial fibrillation, cardio-oncological complications, and inflammatory-related disease. ${ }^{13}$ It has been postulated that the PET assessments of CFR could be helpful, not only for evaluating the need of myocardial revascularization, as that strengthened by Akil et al., ${ }^{1}$ but also for assessing the effects of emerging anti-inflammatory, extreme lipid-lowering, and neurohormonal-modulating therapies. $^{13}$

As a result, the PET-based determination of CFR may be helpful for identifying those patients that are the most likely to benefit from myocardial revascularization, in keeping with conventional recommendations (Table 1), and with the exclusion of the coronary territories with normal CFR and for which a significant CFR improvement is highly unlikely after revascularization. ${ }^{1}$ However, this PET determination also constitutes a tool of choice for better understanding and treating diseases affecting the microvasculature of the heart. Such microvascular diseases are indeed increasingly considered to have a major impact on the prognosis and quality of life of our patients, even when unassociated with any significant obstruction of the epicardial coronary arteries.

\section{Disclosure}

Pierre-Yves Marie and Alain Manrique declares that they have no conflict of interest.

\section{References}

1. Akil S, Hedeer F, Oddstig J, Olsson T, Jögi J, Erlinge D, et al. Appropriate coronary revascularization can be accomplished if myocardial perfusion is quantified by positron emission tomography prior to treatment decision. J Nucl Cardiol 2019. https://doi. org/10.1007/s12350-019-01938-y.
2. Sanchez CE, Dota A, Badhwar V, Kliner D, Smith AJ, Chu D, et al. Revascularization heart team recommendations as an adjunct to appropriate use criteria for coronary revascularization in patients with complex coronary artery disease. Catheter Cardiovasc Interv 2016;88:E103-12.

3. Neumann FJ, Sousa-Uva M, Ahlsson A, Alfonso F, Banning AP, Benedetto U, et al. 2018 ESC/EACTS Guidelines on myocardial revascularization. Eur Heart J 2019;40:87-165.

4. Lin GA, Dudley RA, Redberg RF. Cardiologists' use of percutaneous coronary interventions for stable coronary artery disease. Arch Intern Med 2007;167:1604-9.

5. Kursehi F, Jones PG, Buchanan DM, Abdallah MS, Spertus JA. Variation in patients' perceptions of elective percutaneous coronary intervention in stable coronary artery disease: Cross sectional study. BMJ 2014;349:g5309.

6. Lin GA, Dudley RA, Lucas FL, Malenka DJ, Vittinghoff E, Redberg RF. Frequency of stress testing to document ischemia prior to elective percutaneous coronary intervention. JAMA 2008;300:1765-73.

7. Patel MR, Calhoon JH, Dehmer GJ, Grantham JA, Maddox TM, Maron DJ, et al. ACC/AATS/AHA/ASE/ASNC/SCAI/SCCT/STS 2017 appropriate use criteria for coronary revascularization in patients with stable ischemic heart disease: A Report of the American College of Cardiology Appropriate Use Criteria Task Force, American Association for Thoracic Surgery, American Heart Association, American Society of Echocardiography, American Society of Nuclear Cardiology, Society for Cardiovascular Angiography and Interventions, Society of Cardiovascular Computed Tomography, and Society of Thoracic Surgeons. J Nucl Cardiol 2017;24:1759-92.

8. Tonino PA, Fearon WF, De Bruyne B, Oldroyd KG, Leesar MA, Ver Lee PN, et al. Angiographic versus functional severity of coronary artery stenoses in the FAME study fractional flow reserve versus angiography in multivessel evaluation. J Am Coll Cardiol 2010;55:2816-21.

9. Bateman TM, Dilsizian V, Beanlands RS, DePuey EG, Heller GV, Wolinsky DA. American Society of Nuclear Cardiology and Society of Nuclear Medicine and Molecular Imaging Joint Position Statement on the clinical indications for myocardial perfusion PET. J Nucl Cardiol 2016;23:1227-31.

10. Otaki Y, Manabe O, Miller RJH, Manrique A, Nganoa C, Roth N, et al. Quantification of myocardial blood flow by CZT-SPECT with motion correction and comparison with ${ }^{15} \mathrm{O}$-water PET. J Nucl Cardiol 2019. https://doi.org/10.1007/s12350-019-01854-1.

11. Agostini D, Roule V, Nganoa C, Roth N, Baavour R, Parienti JJ, et al. First validation of myocardial flow reserve assessed by dynamic 99mTc-sestamibi CZT-SPECT camera: Head to head comparison with ${ }^{15} \mathrm{O}$-water PET and fractional flow reserve in patients with suspected coronary artery disease The WATERDAY study. Eur J Nucl Med Mol Imaging 2018;45:1079-90.

12. Danad I, Raijmakers PG, Driessen RS, Leipsic J, Raju R, Naoum $\mathrm{C}$, et al. Comparison of coronary CT angiography, SPECT, PET, and hybrid imaging for diagnosis of ischemic heart disease determined by fractional flow reserve. JAMA Cardiol 2017;2:1100-7.

13. Taqueti VR, Di Carli MF. Clinical significance of noninvasive coronary flow reserve assessment in patients with ischemic heart disease. Curr Opin Cardiol 2016;31:662-9.

Publisher's Note Springer Nature remains neutral with regard to jurisdictional claims in published maps and institutional affiliations. 